\title{
Horizon quantum fuzziness for non-singular black holes
}

\author{
Andrea Giugno $^{1, \mathrm{a}} \mathbb{D}$, Andrea Giusti ${ }^{1,2,3, \mathrm{~b}}$, Alexis Helou ${ }^{1, \mathrm{c}}$ \\ ${ }^{1}$ Arnold Sommerfeld Center, Ludwig-Maximilians-Universität, Theresienstraße 37, 80333 Munich, Germany \\ ${ }^{2}$ Dipartimento di Fisica e Astronomia, Università di Bologna, Via Irnerio 46, 40126 Bologna, Italy \\ ${ }^{3}$ I.N.F.N., Sezione di Bologna, IS-FLAG, Via B. Pichat 6/2, 40127 Bologna, Italy
}

Received: 10 January 2018 / Accepted: 5 March 2018 / Published online: 13 March 2018

(C) The Author(s) 2018

\begin{abstract}
We study the extent of quantum gravitational effects in the internal region of non-singular, Hayward-like solutions of Einstein's field equations according to the formalism known as horizon quantum mechanics. We grant a microscopic description to the horizon by considering a huge number of soft, off-shell gravitons, which superimpose in the same quantum state, as suggested by Dvali and Gomez. In addition to that, the constituents of such a configuration are understood as loosely confined in a binding harmonic potential. A simple analysis shows that the resolution of a central singularity through quantum physics does not tarnish the classical description, which is bestowed upon this extended self-gravitating system by General Relativity. Finally, we estimate the appearance of an internal horizon as being negligible, because of the suppression of the related probability caused by the large number of virtual gravitons.
\end{abstract}

\section{Introduction}

The theory of General Relativity (GR) still presents some major physical problems, despite having maintained a high degree of consistency with astrophysical observations for more than a century. One of such issues arises in a merely classical framework. In fact, the existence of a trapped surface implies geodesic incompleteness in a globally hyperbolic system, which is solution of Einstein's field equations sourced by some reasonable matter distribution (e.g. one that obeys the null energy condition). This is the content of the so-called Singularity theorems in a nutshell: nothing prevents the formation of a curvature singularity at some stage of the gravitational collapse [1], at least in the spheri-

\footnotetext{
a e-mail: A.Giugno@physik.uni-muenchen.de

be-mail: agiusti@bo.infn.it

c e-mail: alexis.helou@physik.uni-muenchen.de
}

cally symmetric case. ${ }^{1}$ One may logically argue that this setback stems from the failure of classical physics at describing short-scale phenomena, for which a fully quantum apparatus is required. Unfortunately, any attempt at quantising GR bumps inevitably into various inconsistencies, which lead to the infamous UV-incompleteness of the theory (for more information, see e.g. [3] and references therein).

A sensible argument to tackle down this premise is to assume that our way of modeling gravity should be extensively modified, as we get closer and closer to regions of extreme curvature. In view of this statement, GR should be regarded as a low-energy effective theory of gravity, which loses its reliability beyond a certain energy scale. Therefore, it is of paramount importance to study the extent of such corrections, for they can provide some hints at a bottom-up construction of a self-consistent quantum theory of gravity.

One of the main ideas emerging from almost every tentative model of quantum gravity (QG) is the appearance of a critical energy scale $\Lambda$ (and a corresponding length scale ${ }^{2}$ $\ell \sim \hbar / \Lambda$ ) above which the quantum nature of gravity cannot be completely neglected any longer. In other words, one expects quantum gravitational effects to modify the geometric description of the spacetime, when the scalar curvature $\mathcal{R} \sim \ell^{-2}$. This feature should be accordingly matched by suitable changes in the structure of the metric tensor, as long as the classical metric description is preserved. However, it is important to remark that $\ell$ does not coincide with the QG regime $\ell_{Q G}$, but rather it is just the scale at which some corrections to GR come into play in the form of appropriate effective metric tensors. Here we still assume that $\ell_{Q G} \ll \ell$, in order to limit ourselves to simple modifications of the field

\footnotetext{
1 For the sake of completeness: the singularity theorems tell us nothing about the nature of this singularity (for a recent review on the topic, see [2]), indeed they are not forced to be curvature singularities if we move away from the spherically symmetric case.

2 We write $\hbar=\ell_{\mathrm{P}} m_{\mathrm{P}}$ and $G_{\mathrm{N}}=\ell_{\mathrm{P}} / m_{\mathrm{P}}$ in Planck units. Nonetheless, we assume $c=1$.
} 
equations, while avoiding to deal with full-fledged quantum degrees of freedom of gravity in the process.

The first proposal for a non-singular solution of the field equations of GR equipped with an event horizon dates back to the Sixties, with the seminal paper [4] by Bardeen. As stressed in [5], the Bardeen black hole is an example of a more general class of solutions of the gravitational field equations in which the regular interior is obtained by assuming a vacuum-like equation of state for the matter content below a certain length scale, in strict analogy with the previous discussion. This approach, when applied to static spherically symmetric cases, leads to regular black hole solutions with a de Sitter-like core and makes it reasonable to assume that a proper UV-complete theory of gravity should lead to some physically fundamental constraints on the values of the curvature invariants [6]. It is therefore important to stress that the metric studied here (and proposed by Hayward [7]) actually satisfies the said limitations (see e.g. [8]).

The purpose of this paper is to analyse some semiclassical aspects of the Hayward spacetime by means of the horizon quantum mechanics formalism [9-11], while working within the framework of the corpuscular model for quantum black holes [12-15].

This paper is therefore organised as follows:

In Sects. 2 and 3, we first review some general aspects of the geometry taken into consideration and we introduce all the main operational features of the horizon quantum mechanics.

Consequently, in Sect. 4, we first review the corpuscular model for black holes, together with its connection with harmonic black hole model, and we then compute the probability for $N$ soft, virtual gravitons to be confined either on the internal or the external horizon.

Finally, we conclude the paper with remarks and hints for future research.

\section{Geometry of Hayward's solution}

A pivotal example of static non-singular black hole, belonging to the class of solutions of the Einstein's equations discussed above, is the so-called Hayward spacetime, defined by the line element

$d s^{2}=-f(r) d v^{2}+2 d v d r+r^{2} d \Omega^{2}$,

with

$f(r)=1-\frac{2 \ell_{\mathrm{P}} M r^{2} / m_{\mathrm{P}}}{r^{3}+2 \ell_{\mathrm{P}} M \ell^{2} / m_{\mathrm{P}}}$.

In the above, $v$ is the advanced time coordinate, $r$ the areal radius, $d \Omega^{2}=d \theta^{2}+\sin ^{2} \theta d \varphi$ the volume element of a 2 sphere, $M$ the ADM mass and $\ell$ represents the critical parameter arising from the corresponding UV-complete theory of gravity. In particular, this model is built in such a way that we recover the asymptotic fall off of the Schwarzschild metric as $r \rightarrow \infty$ and a "de Sitter-like" behavior

$f(r) \sim 1-\frac{r^{2}}{\ell^{2}}, \quad$ as $r \rightarrow 0$.

In addition to that, the corresponding Einstein's field equations reduce to a simple form, $G_{\mu \nu} \sim-\left(3 / \ell^{2}\right) g_{\mu \nu}$, near the origin. For further details on the topic, we invite the interested reader to refer to $[7,8]$.

The trapping horizons of the Hayward spacetime are given by the zeros of the equation $\nabla_{\mu} r \nabla^{\mu} r=0$, that is by the ones of the function $f(r)$ in Eq. (2). This equation reads

$r^{3}-2 \frac{\ell_{\mathrm{P}} M}{m_{\mathrm{P}}} r^{2}+2 \frac{\ell_{\mathrm{P}} M}{m_{\mathrm{P}}} \ell^{2}=0$.

In particular, in the following we will be interested in those configurations that allow for the formation of two distinct horizons (such as in the Kerr or Reissner-Nordström cases for example).

Now, the left hand side of Eq. (4) is a cubic polynomial. The fundamental theorem of algebra states that it will admit three (generally) complex roots, which we can denote by $R_{\mathrm{H}}^{>}$, $R_{\mathrm{H}}^{-}$and $R_{\mathrm{H}}^{<}$. Then, from Vieta's theorem we get the following relations

$$
\begin{aligned}
& R_{\mathrm{H}}^{>}+R_{\mathrm{H}}^{-}+R_{\mathrm{H}}^{<}=\frac{2 \ell_{\mathrm{P}} M}{m_{\mathrm{P}}}>0, \\
& R_{\mathrm{H}}^{>} R_{\mathrm{H}}^{-}+R_{\mathrm{H}}^{>} R_{\mathrm{H}}^{<}+R_{\mathrm{H}}^{-} R_{\mathrm{H}}^{<}=0, \\
& R_{\mathrm{H}}^{>} R_{\mathrm{H}}^{-} R_{\mathrm{H}}^{<}=2 \frac{\ell_{\mathrm{P}} M}{m_{\mathrm{P}}} \ell^{2}<0 .
\end{aligned}
$$

If $R_{\mathrm{H}}^{>}, R_{\mathrm{H}}^{-}, R_{\mathrm{H}}^{<} \in \mathbb{R}$, it is easy to infer from Eq. (7) that either one or all of the roots are negative, whereas Eq. (5) forbids the latter possibility. Therefore, if the three roots are real, one must have two positive roots and a negative one. If this is the case, we then denote the negative solution with $R_{\mathrm{H}}^{-}$ and the other two with $R_{\mathrm{H}}^{>}, R_{\mathrm{H}}^{<}$according to the condition $R_{\mathrm{H}}^{>} \geq R_{\mathrm{H}}^{<}$.

If we perform the change of variable $z=r-\left(2 \ell_{\mathrm{P}} M / 3 m_{\mathrm{P}}\right)$ in Eq. (4) we get

$z^{3}-\frac{4 \ell_{\mathrm{P}}^{2} M^{2}}{3 m_{\mathrm{P}}^{2}} z-\frac{16 \ell_{\mathrm{P}}^{3} M^{3}}{27 m_{\mathrm{P}}^{3}}+\frac{2 \ell_{\mathrm{P}} M}{m_{\mathrm{P}}} \ell^{2}=0$,

to which we can then apply the Cardan method for solving cubic equations.

The discriminant is

$\Delta=\left(\frac{2 \ell_{\mathrm{P}} M}{m_{\mathrm{P}}} \ell\right)^{2}\left(\frac{16 \ell_{\mathrm{P}}^{2} M^{2}}{m_{\mathrm{P}}^{2}}-27 \ell^{2}\right)$,

so that the equation admits at least two distinct real solutions when it is strictly positive, and two degenerate real solutions if it vanishes. Since the coefficients of the polynomial are 
functions of the ADM mass $M$ and the cut-off $\ell$, the horizon structure is regulated by a simple inequality, namely

$0 \leq \frac{3 \sqrt{3} m_{\mathrm{P}}}{4 \ell_{\mathrm{P}}} \ell \leq M$.

It is worth noting that the saturation of the r.h.s. inequality represents the extremal case, whereas two distinct horizons appear otherwise.

Summarizing, the three solutions are

$$
\begin{aligned}
R_{\mathrm{H}}^{(j)}= & \frac{4 \ell_{\mathrm{P}} M}{3 m_{\mathrm{P}}} \cos \left[\frac{1}{3} \arccos \left(1-\frac{27 m_{\mathrm{P}}^{2} \ell^{2}}{8 \ell_{\mathrm{P}}^{2} M^{2}}\right)+\frac{2 \pi j}{3}\right] \\
& +\frac{2 \ell_{\mathrm{P}} M}{3 m_{\mathrm{P}}}, \quad j=1,2,3 .
\end{aligned}
$$

Thanks to Eq. (10), $R_{\mathrm{H}}^{(1)}$ ranges from $-\frac{2 \ell_{\mathrm{P}} M}{3 m_{\mathrm{P}}}$ to $0^{-}$, allowing the identification with $R_{\mathrm{H}}^{-}$. The same procedure reveals that $j=2$ represents $R_{\mathrm{H}}^{<}$, since its lower bound is 0 , whence $j=3$ gives $R_{\mathrm{H}}^{>}$, that can grow up to $2 \ell_{\mathrm{P}} M / m_{\mathrm{P}}$. Besides, the extremal case corresponds to a value of the radius given by

$R_{\mathrm{H}, \text { ext }}=\frac{4 \ell_{\mathrm{P}} M}{3 m_{\mathrm{P}}}$,

which is obtained by setting $R_{\mathrm{H}}^{<}=R_{\mathrm{H}}^{>}$.

When the internal cut-off $\ell$ is such that

$\ell \ll \frac{\ell_{\mathrm{P}} M}{m_{\mathrm{P}}}$,

the metric function (2) approximatively describes a Schwarzschild solution with a small, but (potentially) still macroscopic, inner de Sitter core. In fact, if we recall that

$$
\begin{aligned}
\arccos \left(1-\frac{27}{8} x^{2}\right) & =\frac{3 \sqrt{3}}{2} x+\mathcal{O}\left(x^{3}\right), \\
\cos x & =1-\frac{x^{2}}{2}+\mathcal{O}\left(x^{4}\right),
\end{aligned}
$$

for $x \ll 1$, then expanding Eq. (11) for $x=m_{\mathrm{P}} \ell / \ell_{\mathrm{P}} M \ll 1$ it is easy to see that

$$
\begin{aligned}
& R_{\mathrm{H}}^{>} \sim \frac{2 \ell_{\mathrm{P}}}{m_{\mathrm{P}}} M-\frac{m_{\mathrm{P}} \ell^{2}}{2 \ell_{\mathrm{P}} M}=R_{\mathrm{s}}-\frac{\ell^{2}}{R_{\mathrm{S}}}, \\
& R_{\mathrm{H}}^{<} \sim \ell+\frac{m_{\mathrm{P}} \ell^{2}}{4 \ell_{\mathrm{P}} M}=\ell+\frac{\ell^{2}}{2 R_{\mathrm{s}}},
\end{aligned}
$$

where $R_{\mathrm{S}} \equiv 2 \ell_{\mathrm{P}} M / m_{\mathrm{P}}$ is the Schwarzschild radius.

\section{Horizon quantum mechanics}

In this section, we shall introduce the reader to some general aspects of the horizon quantum mechanics (HQM), according to its most recent updates [16-22].

If one envisages the source of the gravitational field as a purely quantum object under this perspective, then one can faithfully picture both the ADM mass and the gravitational radius as quantum variables in return.

It is therefore reasonable to describe the entire system in terms of an Hilbert space $\mathcal{H}$ defined as

$\mathcal{H}=\mathcal{H}_{\mathrm{S}} \otimes \mathcal{H}_{\mathrm{G}}$

where $\mathcal{H}_{\mathrm{S}}$ and $\mathcal{H}_{\mathrm{G}}$ represent the Hilbert spaces for the source and for the geometry, respectively.

The quantum state of the source should be specified in terms of an Hamiltonian operator $\widehat{H}:=\widehat{H} \otimes \widehat{\mathbb{I}}_{\mathrm{G}}$, with $\widehat{\mathbb{I}}_{\mathrm{G}}$ the identity on $\mathcal{H}_{\mathrm{G}}$, such that

$\widehat{H}=\left(\sum_{\alpha} E_{\alpha}\left|E_{\alpha}\right\rangle\left\langle E_{\alpha}\right|\right) \otimes \widehat{\mathbb{I}}_{\mathrm{G}}$,

where $\left|E_{\alpha}\right\rangle$ represent the "energy" eigenstates of the source corresponding to the eigenvalues $E_{\alpha}$ and $\alpha$ is a set of quantum numbers parametrising its spectral decomposition.

In this framework [11,21,22], we depict the wave-function of the whole system $|\Psi\rangle \in \mathcal{H}$ as an entangled state among $\left|E_{\alpha}\right\rangle$ and the eigenstates of the gravitational radius operators acting on $\mathcal{H}_{\mathrm{G}}$, i.e.

$$
|\Psi\rangle=\sum_{\alpha, \beta, \gamma} C\left(E_{\alpha}, R_{\mathrm{H}_{\beta}}>R_{\mathrm{H}_{\gamma}}^{<}\right)\left|E_{\alpha}\right\rangle|\beta\rangle_{>}|\gamma\rangle_{<},
$$

where

$\widehat{R}_{\mathrm{H}}^{(j)}|\beta\rangle_{(j)}=R_{\mathrm{H}_{\beta}}^{(j)}|\beta\rangle_{(j)}$,

with the subscript " $j$ " standing for either " $>$ " or " $<$ ".

If we now connect the quantum uplifting of the ADM mass with the spectral decomposition (18), it is easy to see that not all the vectors belonging to $\mathcal{H}$ are physical states. In fact, they are the ones that allow us to gain a horizon-law relation, intended as a constraint "à la Gupta-Bleuler", between the spectrum of $\widehat{H}$ and the one of $\widehat{R}_{\mathrm{H}}^{(j)}$, with $j \in\{>,<\}$, namely

$\left(\widehat{R}_{\mathrm{H}}^{>}-\widehat{\mathcal{O}}^{>}\right)|\Psi\rangle_{\text {phys }}=0$,

$\left(\widehat{R}_{\mathrm{H}}^{<}-\widehat{\mathcal{O}}^{<}\right)|\Psi\rangle_{\text {phys }}=0$.

In this case, the operators $\widehat{\mathcal{O}}^{<}$and $\widehat{\mathcal{O}}^{>}$are chosen in order to reproduce the approximated relations in Eqs. (15) and (16), i.e.

$\widehat{\mathcal{O}}^{>}=\frac{2 \ell_{\mathrm{P}}}{m_{\mathrm{P}}} \widehat{H}-\frac{m_{\mathrm{P}} \ell^{2}}{2 \ell_{\mathrm{P}}} \widehat{H}^{-1}$,

$\hat{\mathcal{O}}^{<}=\ell+\frac{m_{\mathrm{P}} \ell^{2}}{4 \ell_{\mathrm{P}}} \widehat{H}^{-1}$.

Notice that $\ell$ is regarded as an external parameter. This is reasonable because it should represent some sort of UV cutoff arising from a fundamental theory of quantum gravity rather than from a semiclassical description of the interplay between the quantum source and the geometry of the system. 
Now, if we trace away from $|\Psi\rangle$ the contribution of the geometry, we are left with the wave-function of the collapsed matter, i.e.

$|\Psi\rangle_{\mathrm{S}}=\sum_{\alpha} C_{\mathrm{S}}\left(E_{\alpha}\right)\left|E_{\alpha}\right\rangle$,

$C_{\mathrm{S}}\left(E_{\alpha}\right) \equiv C\left(E_{\alpha}, R_{\mathrm{H}}>\left(E_{\alpha}\right), R_{\mathrm{H}}<\left(E_{\alpha}\right)\right)$,

where the relations $R_{\mathrm{H}}>\left(E_{\alpha}\right)$ and $R_{\mathrm{H}}<\left(E_{\alpha}\right)$ are provided by the constraints (21) and (22). This quantum state is then related to the classical ADM mass through the expectation value

$M \mapsto\left\langle\Psi_{\mathrm{S}}|\widehat{H}| \Psi_{\mathrm{S}}\right\rangle=\sum_{\alpha}\left|C_{\mathrm{S}}\left(E_{\alpha}\right)\right|^{2} E_{\alpha}$.

By tracing out the contribution of the source, together with one of the two contributions of the horizons, we can then define two (non-normalized) horizon wave-functions, namely

$\Psi_{>}\left(R_{\mathrm{H}}^{>}\right) \simeq C\left(E_{\alpha}\left(R_{\mathrm{H}}^{>}\right), R_{\mathrm{H}}^{>}, R_{\mathrm{H}}<\left(R_{\mathrm{H}}^{>}\right)\right)$,

$\Psi_{<}\left(R_{\mathrm{H}}^{<}\right) \simeq C\left(E_{\alpha}\left(R_{\mathrm{H}}^{<}\right), R_{\mathrm{H}}^{>}\left(R_{\mathrm{H}}^{<}\right), R_{\mathrm{H}}^{<}\right)$.

Then, from these two wave-functions we can build two probability density functions for a lump of matter $|\Psi\rangle_{\mathrm{S}}$ to be equipped with an internal horizon located in $R_{\mathrm{H}}^{<}$and an external one in $R_{\mathrm{H}}^{>}$, once gravitational collapse has taken place. To be more precise, we have

$\mathcal{P}_{>}\left(r_{\mathrm{H}}^{>}\right):=4 \pi r_{\mathrm{H}}^{>2}\left|\Psi_{>}\left(r_{\mathrm{H}}^{>}\right)\right|^{2}$,

$\mathcal{P}_{<}\left(r_{\mathrm{H}}^{<}\right):=4 \pi r_{\mathrm{H}}^{<2}\left|\Psi_{<}\left(r_{\mathrm{H}}^{<}\right)\right|^{2}$.

This allows us to infer whether $|\Psi\rangle_{\mathrm{S}}$ sources a black hole. Indeed, denoting the probability density for $|\Psi\rangle_{\mathrm{S}}$ to be localized within the external horizon,

$\wp\left(r<r_{\mathrm{H}}^{>}\right)=P_{\mathrm{S}}\left(r<r_{\mathrm{H}}^{>}\right) \mathcal{P}_{>}\left(r_{\mathrm{H}}^{>}\right)$,

where $P_{\mathrm{S}}\left(r<r_{\mathrm{H}}^{>}\right)=4 \pi \int_{0}^{r_{\mathrm{H}}^{>}} \bar{r}^{2}\left|\Psi_{\mathrm{S}}(\bar{r})\right|^{2} \mathrm{~d} \bar{r}$, then the probability for $|\Psi\rangle_{\mathrm{S}}$ to be a black hole is given by

$P_{\mathrm{BH}}=\int_{0}^{\infty} \wp\left(r<r_{\mathrm{H}}^{>}\right) \mathrm{d} r_{\mathrm{H}}^{>}$.

In a similar fashion, we can estimate the likelihood for the system to be trapped within the internal horizon. It will be denoted by $P_{\mathrm{IH}}$.

\section{Application to non-singular BHs}

Before investigating whether the effective inclusion of QG effects in the interior of an event horizon preserves or spoils the GR description of gravitating systems, we have to model a suitable microscopic theory. Therefore, we quickly recall the basic features of the corpuscular black hole model of
Dvali and Gomez [12-15,23-25], which is the setting we are taking into consideration. In this way, black holes are extended objects, which hint at a UV completion of gravity through the production of a huge number $N$ of soft, gapless modes, instead of a low amount of very hard ones.

By means of a binding potential, these constituents can superimpose in one particular quantum state, effectively making the compact object a self-gravitating Bose-Einstein condensate (BEC) [26-33], at least to leading order of approximation. Moreover, the virtual gravitons are expected to be marginally bound in this potential well, giving rise to the so-called maximal packing relation

$\mu+U_{\mathrm{N}} \simeq 0$

expressed in terms of the Newtonian potential energy profile

$U_{\mathrm{N}} \simeq-N \alpha \mu \Theta\left(\lambda_{\mu}-r\right)$,

where $\mu$ is the graviton effective mass, related to its Compton/de Broglie wavelength by $\lambda_{\mu} \simeq m_{\mathrm{P}} \ell_{\mathrm{P}} / \mu$, and $\alpha=$ $\ell_{\mathrm{P}}^{2} / \lambda_{\mu}^{2}=\mu^{2} / m_{\mathrm{P}}^{2}$ is the effective coupling constant.

This set-up is particularly interesting since we can quantize the relevant features of a black hole solely in terms of the mean number of constituents $N$. In fact, as one can easily infer from the energy balance (35), the effective coupling reads $\alpha \simeq 1 / N$, while

$M \simeq \sqrt{N} m_{\mathrm{P}}, \quad \mu \simeq \frac{m_{\mathrm{P}}}{\sqrt{N}}$

and $\lambda_{\mu}=\hbar / \mu=\sqrt{N} \ell_{\mathrm{P}}$. In particular, it is also possible to justify the latter estimate through a simple argument. If a quantum black hole is supposed to establish a bridge between quantum mechanics $(\mathrm{QM})$ and $\mathrm{GR}$, the characteristic size of quantum fluctuations, $\lambda_{\mu}$, should match indeed the extent of a typical gravitational radius $R_{g} \sim \ell_{\mathrm{P}} M / m_{\mathrm{P}}$. Moreover, in these terms, the bound (10) can be immediately rewritten as

$\ell \leq \frac{4}{3 \sqrt{3}} \frac{M}{m_{\mathrm{P}}} \ell_{\mathrm{P}}=\frac{4}{3 \sqrt{3}} \sqrt{N} \ell_{\mathrm{P}}$.

Although approximating the binding potential with a square well leads to interesting results [34], a more appropriate approximation for such a potential is provided by the harmonic black hole model [21,35], for which we have that $V(r)=V_{0}(r) \Theta\left(\lambda_{\mu}-r\right)$, where

$V_{0}(r)=\frac{1}{2} \mu \omega^{2}\left(r^{2}-\lambda_{\mu}^{2}\right)$.

We chose to set the width of the well of order $\lambda_{\mu}$ in order to enforce the connection between the confinement given by the Schwarzschild radius and quantum physics. Moreover, the central singularity is resolved by the quantum mechanical properties of collapsed matter, as it has already been discussed by means of post-Newtonian arguments [23,24]. 
Therefore, the relevant quantum states of the toy gravitons in this spherically symmetric geometry are depicted by the single-particle eigenfunctions

$\phi_{n l}(r)=\mathcal{N}_{n l} r^{l} L_{n}^{(l+1 / 2)}\left(\frac{\omega r^{2}}{\lambda_{\mu}}\right) \exp \left(-\frac{\omega r^{2}}{2 \lambda_{\mu}}\right)$,

where $\mathcal{N}_{n l}$ is a normalisation coefficient and $L_{n}^{a}(x)$ are the generalised Laguerre polynomials. These functions are solutions of the Schrödinger equation

$\frac{\ell_{\mathrm{P}}^{2} m_{\mathrm{P}}^{2}}{2 \mu}\left(\Delta-\frac{l(l+1)}{r^{2}}\right) \phi_{n l}(r)=\left(V_{0}-E_{n l}\right) \phi_{n l}(r)$,

under the radial Laplacian in spherical symmetry, i.e.

$\triangle \phi_{n l}(r)=\frac{1}{r^{2}} \frac{d}{d r}\left[r^{2} \frac{d \phi_{n l}(r)}{d r}\right]$.

We recall that the energy spectrum reads

$E_{n l}=\ell_{\mathrm{P}} m_{\mathrm{P}} \omega\left(2 n+l+\frac{3}{2}\right)+V_{0}$,

where $V_{0}=V_{0}(0)$. The maximal quantum numbers $n_{0}$ and $l_{0}$ designate the most loosely bound state, which translates to $E_{n_{0}, l_{0}} \approx 0$. This concept is particularly useful because, from Eq. (38) and the condition

$V_{0}=-\ell_{\mathrm{P}} m_{\mathrm{P}} \omega\left(2 n_{0}+l_{0}+\frac{3}{2}\right)$,

one can immediately read off that

$\omega=\frac{2}{\lambda_{\mu}}\left(2 n_{0}+l_{0}+\frac{3}{2}\right)$.

The energy spectrum (42) is then

$E_{n l}=2 \mu\left(2 n_{0}+l_{0}+\frac{3}{2}\right)\left[2\left(n-n_{0}\right)+\left(l-l_{0}\right)\right]$.

Since the Hayward spacetime describes a static black hole, one can easily show that the (Komar) total angular momentum vanishes. Therefore, we choose to focus on quantum states satisfying $l=l_{0} \equiv 0$.

Although many microscopic configurations may realise this feature, we impose this condition for the sake of simplicity and to avoid the unnatural choice of one particular Clebsch-Gordan coefficient among a great number thereof.

Eventually, the quantum state of every toy graviton is decomposed over the basis spanned by

$$
\begin{aligned}
\phi_{n}(r)= & \mathcal{N}_{n} L_{n}^{(1 / 2)}\left(\frac{\omega r^{2}}{\lambda_{\mu}}\right) \exp \left(-\frac{\omega r^{2}}{2 \lambda_{\mu}}\right) \\
\simeq & \mathcal{N}_{n} L_{n}^{(1 / 2)}\left[\frac{\left(4 n_{0}+3\right) r^{2}}{N \ell_{\mathrm{P}}^{2}}\right] \\
& \times \exp \left[-\left(2 n_{0}+\frac{3}{2}\right) \frac{r^{2}}{N \ell_{\mathrm{P}}^{2}}\right]
\end{aligned}
$$

according to the scaling relation for $\lambda_{\mu}$, Eq. (44) and

$$
\frac{\omega}{\lambda_{\mu}}=\frac{2}{\lambda_{\mu}^{2}}\left(2 n_{0}+\frac{3}{2}\right)=\frac{4 n_{0}+3}{N \ell_{\mathrm{P}}^{2}} \text {. }
$$

\subsection{Black hole probability}

The entire system is described, in terms of the single-particle wave-functions $\phi_{n}^{(i)}$, by the multi-particle state

$$
|\Phi\rangle=\bigotimes_{i=1}^{N}\left|\phi^{(i)}\right\rangle=\bigotimes_{i=1}^{N}\left[\sum_{n=0}^{\infty} c_{n}\left|\phi_{n}^{(i)}\right\rangle\right]
$$

which is an eigenstate of the $N$-particle Hamiltonian according to

$$
\langle\Phi|\widehat{H}| \Phi\rangle=M \text {. }
$$

Let us recall that $M=N \mu$ is just the ADM mass.

Clearly, in position space

$$
\begin{aligned}
\left\langle r \mid \phi_{0}\right\rangle= & \phi_{0}(r) \simeq\left(\frac{4 n_{0}+3}{\pi N \ell_{\mathrm{P}}^{2}}\right)^{3 / 4} \\
& \times \exp \left[-\left(2 n_{0}+\frac{3}{2}\right) \frac{r^{2}}{N \ell_{\mathrm{P}}^{2}}\right] .
\end{aligned}
$$

Since our purpose is the comparison of the result with a singular case, i.e. Schwarzschild $(\ell=0)$, we can afford to model the multi-particle quantum state in the simplest possible way. That is to say, we assume that most of the contribution to the probability densities comes from the harmonic states (39) carrying the smallest $n$, i.e. $c_{n}^{(i)} \sim \delta_{n 0}, \forall i=1, \ldots, N$. Therefore, we can reliably approximate the multi-particle state (48) with the product

$$
|\Phi\rangle \simeq \bigotimes_{i=1}^{N}\left|\phi_{0}^{(i)}\right\rangle=\left[\left|\phi_{0}\right\rangle\right]^{N} .
$$

As discussed in [21], it is possible to estimate the probability for this quantum source to be a black hole by

$P_{\mathrm{BH}}(N)=\int_{0}^{\infty} \wp\left(r_{1}<R_{\mathrm{H}}, \ldots, r_{N}<R_{\mathrm{H}}\right) \mathrm{d} R_{\mathrm{H}}$,

in a simple way. In fact, from Eq. (51) it is easy to see that

$$
P_{\mathrm{S}}\left(r_{1}<R_{\mathrm{H}}, \ldots, r_{N}<R_{\mathrm{H}}\right)=\prod_{i=1}^{N} P_{\mathrm{S}}\left(r_{i}<R_{\mathrm{H}}\right)
$$

and the horizon wave-function for the outer horizon gives rise to a delta-shaped total probability density, peaked on the corresponding expectation value

$\mathcal{P}_{\mathrm{H}}^{>}\left(R_{\mathrm{H}}\right) \simeq \delta\left(R_{\mathrm{H}}-\left\langle\widehat{R}_{\mathrm{H}}^{>}\right\rangle\right)$.

This last estimate follows from the fact that we consider black holes of astrophysical size, which means that $N$ is a huge number and the uncertainty over the horizon radius is 
negligible, from a collective point of view, contrarily to the single-particle case analysed in e.g. [9].

The fact that the vast majority of the toy gravitons is sharply distributed, at least from a macroscopic perspective, has a simple consequence on the expectation value of the horizon operators (23) and (24). Since the cut-off $\ell$ is a non-operatorial quantity, coming from an effective quantumgravitational description, with the aid of Eq. (49) it is straightforward to infer $\left\langle\widehat{R}_{\mathrm{H}}^{>}\right\rangle=R_{\mathrm{H}}^{>}$once the constraint (21) has been implemented. Obviously, the same holds for $\left\langle\hat{R}_{\mathrm{H}}^{<}\right\rangle$.

The sought probability reads

$P_{\mathrm{BH}}(\ell ; N)=\prod_{i=1}^{N} P_{\mathrm{S}}\left(r_{i}<\left\langle\widehat{R}_{\mathrm{H}}^{>}\right\rangle\right)=\left[P_{\mathrm{S}}\left(r<\left\langle\widehat{R}_{\mathrm{H}}^{>}\right\rangle\right)\right]^{N}$

with a sufficient degree of accuracy.

Taking profit of Eq. (15) and recalling the definition of the lower-incomplete Euler Gamma function

$\gamma(s, x)=\int_{0}^{x} \mathrm{~d} t t^{s-1} e^{-t}$,

we can easily compute

$$
\begin{aligned}
P_{\mathrm{S}}\left(r<\left\langle\widehat{R}_{\mathrm{H}}^{>}\right\rangle\right) & =4 \pi \int_{0}^{R_{\mathrm{H}}^{>}} \mathrm{d} r r^{2}\left|\phi_{0}(r)\right|^{2} \\
& =\frac{2}{\sqrt{\pi}} \gamma\left(\frac{3}{2}, \frac{4 n_{0}+3}{N} \frac{\left(R_{\mathrm{H}}^{>}\right)^{2}}{\ell_{\mathrm{P}}^{2}}\right) \\
& =\frac{2}{\sqrt{\pi}} \gamma\left[\frac{3}{2}, \frac{4 n_{0}+3}{N}\left(\frac{2 M}{m_{\mathrm{P}}}-\frac{m_{\mathrm{P}}}{2 M} \frac{\ell^{2}}{\ell_{\mathrm{P}}^{2}}\right)^{2}\right]
\end{aligned}
$$

and expand the result to the leading order in the cut-off $\ell$

$$
\begin{aligned}
P_{\mathrm{S}}\left(r<\left\langle\widehat{R}_{\mathrm{H}}^{>}\right\rangle\right)= & \frac{2}{\sqrt{\pi}} \gamma\left[\frac{3}{2}, \frac{4\left(4 n_{0}+3\right)}{N} \frac{M^{2}}{m_{\mathrm{P}}^{2}}\right] \\
& -\frac{8 M}{\sqrt{\pi} m_{\mathrm{P}}}\left(\frac{4 n_{0}+3}{N}\right)^{3 / 2} \\
& \times \exp \left[-\frac{4\left(4 n_{0}+3\right) M^{2}}{N m_{\mathrm{P}}^{2}}\right] \frac{\ell^{2}}{\ell_{\mathrm{P}}^{2}} \\
& +\mathcal{O}\left[\frac{\ell^{3}}{\left(\ell_{\mathrm{P}} M / m_{\mathrm{P}}\right)^{3}}\right] .
\end{aligned}
$$

The total probability (55) is therefore given by

$P_{\mathrm{BH}}(\ell ; N)=\left\{\frac{2}{\sqrt{\pi}} \gamma\left[\frac{3}{2}, \frac{4 n_{0}+3}{N}\left(\frac{2 M}{m_{\mathrm{P}}}-\frac{m_{\mathrm{P}}}{2 M} \frac{\ell^{2}}{\ell_{\mathrm{P}}^{2}}\right)^{2}\right]\right\}^{N}$.

Expanding for $m_{\mathrm{P}} \ell / M \ell_{\mathrm{P}} \ll 1$ (as above) and plugging in the scaling relation $M=\sqrt{N} m_{\mathrm{P}}$, we finally have

$$
\begin{aligned}
P_{\mathrm{BH}}(\ell ; N) \simeq & \left\{\frac{2}{\sqrt{\pi}} \gamma\left[\frac{3}{2}, 4\left(4 n_{0}+3\right)\right]\right\}^{N-1} \\
& \left\{\frac{2}{\sqrt{\pi}} \gamma\left[\frac{3}{2}, 4\left(4 n_{0}+3\right)\right]\right. \\
& \left.-\frac{8\left(4 n_{0}+3\right)^{3 / 2}}{\sqrt{\pi} N} \exp \left[-4\left(4 n_{0}+3\right)\right] \frac{\ell^{2}}{\ell_{\mathrm{P}}^{2}}\right\} .
\end{aligned}
$$

It is interesting to see that the difference

$$
\begin{aligned}
\sigma(\ell)= & \left|P_{\mathrm{BH}}(0 ; N)-P_{\mathrm{BH}}(\ell ; N)\right| \\
\sim & \frac{8\left(4 n_{0}+3\right)^{3 / 2}}{\sqrt{\pi} N}\left[\frac{2}{\sqrt{\pi}} \gamma\left(\frac{3}{2}, 4\left(4 n_{0}+3\right)\right)\right]^{N-1} \\
& \times \exp \left[-4\left(4 n_{0}+3\right)\right] \frac{\ell^{2}}{\ell_{\mathrm{P}}^{2}},
\end{aligned}
$$

signals the breaking of a Schwarzschild-like black hole configuration, due to QG effects at the center, when $\sigma(\bar{\ell}) \sim 1$, for a certain value of the cut-off $\bar{\ell}$, which is nonetheless constrained to respect the bound (37).

Recalling that $\gamma(3 / 2, x)$ is a positive and (strictly) monotonically increasing function on $x>0$ with $\sup _{x>0}\{\gamma(3 / 2, x)\}$ $=\sqrt{\pi} / 2<1$, it is easy to see that

$(2 / \sqrt{\pi}) \gamma\left(\frac{3}{2}, 4\left(4 n_{0}+3\right)\right)<1$

and positive for all (fixed) $n_{0}$. Moreover, from the assumption $m_{\mathrm{P}} \ell / M \ell_{\mathrm{P}} \ll 1$ one can easily infer that

$\frac{\ell}{\ell_{\mathrm{P}}} \ll \frac{M}{m_{\mathrm{P}}} \sim \sqrt{N}$.

Thus, plugging these considerations in Eq. (61), we get

$$
\begin{aligned}
\sigma(\ell) \ll & \frac{8\left(4 n_{0}+3\right)^{3 / 2}}{\sqrt{\pi}}\left[\frac{2}{\sqrt{\pi}} \gamma\left(\frac{3}{2}, 4\left(4 n_{0}+3\right)\right)\right]^{N-1} \\
& \times \exp \left[-4\left(4 n_{0}+3\right)\right] \ll 1
\end{aligned}
$$

for all (fixed) value of $n_{0}$ and $\forall N \gg 1$. Therefore, there are no signals of breakdown of the black hole configuration caused by quantum fluctuations, since $\sigma(\ell) \ll 1$.

Concerning the inner horizon, the same argument translates to

$$
P_{\mathrm{S}}\left(r<\left\langle\widehat{R}_{\mathrm{H}}^{<}\right\rangle\right)=\frac{2}{\sqrt{\pi}} \gamma\left[\frac{3}{2}, \frac{4 n_{0}+3}{N}\left(\frac{\ell}{\ell_{\mathrm{P}}}+\frac{m_{\mathrm{P}} \ell^{2}}{4 \ell_{\mathrm{P}}^{2} M}\right)^{2}\right],
$$

which yields

$P_{\mathrm{IH}}(\ell ; N) \simeq\left\{\frac{2}{\sqrt{\pi}} \gamma\left[\frac{3}{2}, \frac{4 n_{0}+3}{N}\left(\frac{\ell}{\ell_{\mathrm{P}}}+\frac{\ell^{2}}{4 \sqrt{N} \ell_{\mathrm{P}}^{2}}\right)^{2}\right]\right\}^{N}$

Again, because of the condition $m_{\mathrm{P}} \ell / M \ell_{\mathrm{P}} \ll 1$, together with the strict monotonicity of $\gamma(3 / 2, x)$, it is easy to see that

$P_{\mathrm{IH}}(\ell ; N) \ll\left\{\frac{2}{\sqrt{\pi}} \gamma\left[\frac{3}{2}, \frac{25}{16}\left(4 n_{0}+3\right)\right]\right\}^{N} \ll 1$ 
for all (fixed) value of $n_{0}$ and $\forall N \gg 1$. This shows that the probability that our static configuration has an internal horizon is negligible.

In particular, it has been proposed [34] that $N \rightarrow \infty$ is the limit, in which a purely classical geometry shall emerge from the effective description of the corpuscular black hole model. This statement is enforced by the present analysis, since

$$
\lim _{N \rightarrow \infty} P_{\mathrm{IH}}(\ell ; N)=0
$$

means that the center of the inner region is not screened by any horizon, as in the vacuum solutions in GR.

\section{Conclusions and outlook}

In this work, we analysed the possible ramifications of applying HQM to non-singular, static metrics. First of all, an observer outside the trapped region sees little or no deviation from Schwarzschild's geometry, even for what concerns the quantum fluctuations over its characteristic features, although the interior is drastically different. This outcome strongly supports the standard knowledge, according to which the central singularity is an artifact of the incompleteness of GR at describing short-scale gravity and not some strange (and appalling) property of nature. The only information about the purely quantum nature of gravitation is encoded in the cut-off $\ell$ alone, which still leaves the freedom to motivate regularity at the origin with a wide variety of microscopic theories. On the other hand, it is of paramount importance to note that the probability to have an inner horizon (66) is always negligible within the framework of the corpuscular model of black holes. This hints at the fact that the formation of a non-singular black hole of the Haywardtype is rather unlikely in this scheme. A straighforward consequence of this findings is the absence of exotic features of Cauchy horizons, such as the so-called "mass inflation".

The reader should bear in mind, however, that we just considered an already formed self-sustained gravitating system and checked to which extent quantum-gravitational fluctuations can break this configuration. We have not been able to compute the probability to take a lump of baryonic matter and get an event horizon, once it has collapsed in some region of space, e.g. as in Refs. [9, 10]. Unfortunately, it is indeed impossible to replicate this machinery in any multi-particle model without requiring a fully dynamical approach, which extends the idea of Ref. [17] including somehow the quantum details of gravitational collapse. This colossal assignment is left for future research.

Acknowledgements A. Giugno and AH were supported by the ERC Advanced Grant 339169 "Selfcompletion", during the realisation of this paper. A. Giusti is partially supported by the INFN Grant FLAG. The research activity of A. Giugno and A. Giusti has also been carried out in the framework of activities of the National Group of Mathematical Physics (GNFM, INdAM).

Open Access This article is distributed under the terms of the Creative Commons Attribution 4.0 International License (http://creativecomm ons.org/licenses/by/4.0/), which permits unrestricted use, distribution, and reproduction in any medium, provided you give appropriate credit to the original author(s) and the source, provide a link to the Creative Commons license, and indicate if changes were made. Funded by $\mathrm{SCOAP}^{3}$.

\section{References}

1. J.R. Oppenheimer, H. Snyder, On continued gravitational contraction. Phys. Rev. 56, 455 (1939)

2. D. Garfinkle, J.M.M. Senovilla, The 1965 Penrose singularity theorem. Class. Quantum Gravity 32(12), 124008 (2015)

3. D. Malafarina, Classical collapse to black holes and quantum bounces: a review. Universe 3(2), 48 (2017)

4. J.M. Bardeen, Non-singular General-Relativistic Gravitational Collapse. in Proceedings of International Conference GR5, Tbilisi University Press, 1968, p. 174

5. S. Ansoldi, Spherical black holes with regular center: a review of existing models including a recent realization with Gaussian sources. arXiv:0802.0330 [gr-qc]

6. V.P. Frolov, M.A. Markov, V.F. Mukhanov, Black holes as possible sources of closed and semiclosed worlds. Phys. Rev. D 41, 383 (1990)

7. S.A. Hayward, Formation and evaporation of non-singular black holes. Phys. Rev. Lett. 96, 031103 (2006)

8. V.P. Frolov, Notes on non-singular models of black holes. Phys. Rev. D 94, 104056 (2016)

9. R. Casadio, Localised particles and fuzzy horizons: a tool for probing quantum black holes. arXiv:1305.3195 [gr-qc]

10. R. Casadio, A.A. Giugnond, O. Micu, Horizon quantum mechanics: a Hitchhiker's guide to quantum black holes. Int. J. Mod. Phys. D 25(02), 1630006 (2016)

11. R. Casadio, A.A. Giugnond, A. Giusti, Global and local horizon quantum mechanics. Gen. Relativ. Gravit. 49(2), 32 (2017)

12. G. Dvali, G.F. Giudice, C. Gomez, A. Kehagias, UV-completion by classicalization. JHEP 1108, 108 (2011)

13. G. Dvali, C. Gomez, Black hole's quantum N-portrait. Fortsch. Phys. 61, 742 (2013)

14. G. Dvali, C. Gomez, Black hole's $1 / N$ hair. Phys. Lett. B 719, 419 (2013)

15. G. Dvali, C. Gomez, Black holes as critical point of quantum phase transition. Eur. Phys. J. C 74, 2752 (2014)

16. R. Casadio, F. Scardigli, Horizon wave-function for single localized particles: GUP and quantum black hole decay. Eur. Phys. J. C 74(1), 2685 (2014)

17. R. Casadio, Horizons and non-local time evolution of quantum mechanical systems. Eur. Phys. J. C 75(4), 160 (2014)

18. R. Casadio, O. Micu, P. Nicolini, Minimum length effects in black hole physics. Fundam. Theor. Phys. 178, 293 (2014)

19. R. Casadio, O. Micu, D. Stojkovic, Inner horizon of the quantum Reissner-Nordström black holes. JHEP 1505, 096 (2015)

20. R. Casadio, O. Micu, D. Stojkovic, Horizon wave-function and the quantum cosmic censorship. Phys. Rev. B 747, 68 (2015)

21. R. Casadio, A. Giugno, A. Giusti, O. Micu, Horizon quantum mechanics of rotating black holes. Eur. Phys. J. C 77(5), 322 (2017)

22. A. Giusti, Horizon quantum mechanics: spherically symmetric and rotating sources. arXiv:1709.10348 [gr-qc] 
23. R. Casadio, A. Giugno, A. Giusti, Matter and gravitons in the gravitational collapse. Phys. Lett. B 763, 337 (2016)

24. R. Casadio, A. Giugno, A. Giusti, M. Lenzi, Quantum corpuscular corrections to the Newtonian potential. Phys. Rev. D 96(4), 044010 (2017)

25. R. Casadio, A. Giugno, A. Giusti, Corpuscular slow-roll inflation. arXiv: 1708.09736 [gr-qc]

26. R. Ruffini, S. Bonazzola, Systems of self-gravitating particles in general relativity and the concept of an equation of state. Phys. Rev. 187, 1767 (1969)

27. M. Colpi, S.L. Shapiro, I. Wasserman, Boson stars: gravitational equilibria of self-interacting scalar fields. Phys. Rev. Lett. 57, 2485 (1986)

28. M. Membrado, J. Abad, A.F. Pacheco, J. Sanudo, Newtonian boson spheres. Phys. Rev. D 40, 2736 (1989)

29. P.-H. Chavanis, T. Harko, Bose-Einstein condensate general relativistic stars. Phys. Rev. D 86, 064011 (2012)
30. F. Kühnel, Bose-Einstein condensates with derivative and longrange interactions as set-ups for analog black holes. Phys. Rev. D 90, 084024 (2104)

31. F. Kühnel, B. Sundborg, High-energy gravitational scattering and Bose-Einstein condensates of gravitons. JHEP 12, 016 (2014)

32. F. Kühnel, B. Sundborg, Decay of graviton condensates and their generalizations in arbitrary dimensions. Phys. Rev. D 90, 064025 (2014)

33. F. Cunillera, C. Germani, The Gross-Pitaevskii equations of a static and spherically symmetric condensate of gravitons. arXiv: 1711.01282 [gr-qc]

34. R. Casadio, A. Giugno, O. Micu, A. Orlandi, Thermal BEC black holes. Entropy 17, 6893 (2015)

35. R. Casadio, A. Orlandi, Quantum harmonic black holes. JHEP 1308, 025 (2103) 